\title{
Two-level approach for solving the inverse problem of defects identification in Eddy Current Testing - type NDT
}

\author{
Piotr PuteK $^{1}$, Guillaume Crevecoeur ${ }^{2}$, Marian SlodičKa ${ }^{1}$ \\ KONSTANTY M. GAWRYLCZYK ${ }^{3}$, ROGER VAN KEER ${ }^{1}$, LUC DUPRÉ \\ ${ }^{1} \mathrm{NaM}^{2}$ Research Group, Department of Mathematical Analysis \\ Ghent University \\ B-9000, Ghent, Belgium \\ e-mail:Piotr.Putek@Ugent.be \\ ${ }^{2}$ Department of Electrical Energy, Systems and Automation \\ Ghent University \\ B-9000 Ghent, Belgium \\ ${ }^{3}$ Department of Electrotechnology and Diagnostic \\ West Pomeranian University of Technology in Szczecin \\ 70-313 Szczecin, Poland
}

(Received: 02.07.2011, revised: 28.09.2011)

\begin{abstract}
This work deals with the inverse problem associated to 3D crack identification inside a conductive material using eddy current measurements. In order to accelerate the time-consuming direct optimization, the reconstruction is provided by the minimization of a last-square functional of the data-model misfit using space mapping (SM) methodology. This technique enables to shift the optimization burden from a time consuming and accurate model to the less precise but faster coarse surrogate model. In this work, the finite element method (FEM) is used as a fine model while the model based on the volume integral method (VIM) serves as a coarse model. The application of the proposed method to the shape reconstruction allows to shorten the evaluation time that is required to provide the proper parameter estimation of surface defects.
\end{abstract}

Key words: defect characterization, electromagnetic inverse problem, two level optimization algorithms, eddy current testing method, sensitivity analysis

\section{Introduction}

The Eddy Current Testing (ECT) type non destructive testing (NDT) is mainly of interest for testing the quality of metallic structures. According to Faraday's law, the capability of a low frequency field produced by a source probe to penetrate the conducting object makes possible to provide the structure recognition during production lines or in-service inspection of

\footnotetext{
* This is extended version of a paper which was presented at the 8th International Conference on Computation in Electronics, Wrocław, 11-14.04.2011.
} 
industrial parts, etc. More precisely, the application of this approach enables to find metal-loss regions produced by corrosion, stress or fatigue, and so on. The perturbation of eddy currents that results from the interaction of an electromagnetic field with one or more defects within conductors, can be observed as a variation in the measurement signal. From this point of view, finding the shape of an examined object or the image of conductivity inside materials based on this signal is just a type of an inverse problem of structure recognition. The voltage or impedance obtained for the multi-frequency of exciting current as well as a different position of the sensor is mostly used as collected data. Hence, this work is addressed to the problem of a 3D bounded void defects reconstruction in a conductive structure arising in ECT-type of NDT. This type of problem appears in many industry branches, therefore, its solution finds a wide application in e.g. atomic energy, automotive, marine manufacturing and aeronautic industry.

For the reconstruction of defects in a conductive object, it is necessary to solve the eddy current inverse problem that is inherently ill-posed and non-linear [1,2]. For the purpose of finding its solution a lot of methods have already been developed. Among these techniques one can find the deterministic and stochastic algorithms, pre-calculated data approach, methods based on the evolution strategy or statistics, linear or quadratic models, artificial neural network or fuzzy-logic, e.g. [3-9].

However, engineering optimization requires highly accurate numerical models, which imply an excessive computational cost, e.g. 3D simulations for complicated geometries. From this point of view, the need exists to speed up the minimization procedure of defects reconstruction arising in the ECT. In this context, the two-level iterative algorithms for solving the eddy current inverse problem are here developed.

Therefore, in the present work, we tackle the inverse problem by combining the Aggressive Space Mapping (ASM) and Manifold Mapping (MM) optimization with Tikhonov regularization technique under the assumption that defects can be approximated by a piecewise conductivity distribution. For the purpose of a coarse model optimization, the regularized GaussNewton iterative method is used. In general, these techniques assume the existence of two models: an expensive, so-called fine model and a coarse model, which is used for generating surrogates that need to approximate the fine model. In this way, the direct optimization of the fine model is replaced by an iterative optimization and an update of the cheaper to calculate but less accurate surrogate models based on the coarse model.

This very efficient, acknowledged engineering technique was not up to now applied for the purpose of defects recognition from the ECT signal. However, recently, this method has become the subject of very intense research in finding a solution of optimization and inverse problems in electromagnetism e.g. [10-12]. From this point of view, its application in the proposed area is promising. The similar inversion methodology was applied to defects characterrization based on Magnetic Flux Leakage measurements [13].

For the numerical verification, the model of ECT system, a variant of which was analyzed in [14], is applied. Moreover, in the presented numerical example it is assumed that the distribution of conductivity in the region of interest, located in a conductive material, can be described using a known function. Note that although the usage of the here proposed SMbased inversion procedure allows considerably shortening the time needed for defect recon- 
struction arising in ECT - type NDT, it is still not the real-time application of defect recognition, which is generally a well-known drawback of the optimization algorithms based on the gradient methods.

\section{Eddy current type testing - type NDT}

The eddy current inspection techniques are non-destructive and contact-less quantitative methods. Among others, its application allows to reconstruct the cracks and flaws in a conductive material placed on the surfaces as well as inside the material.

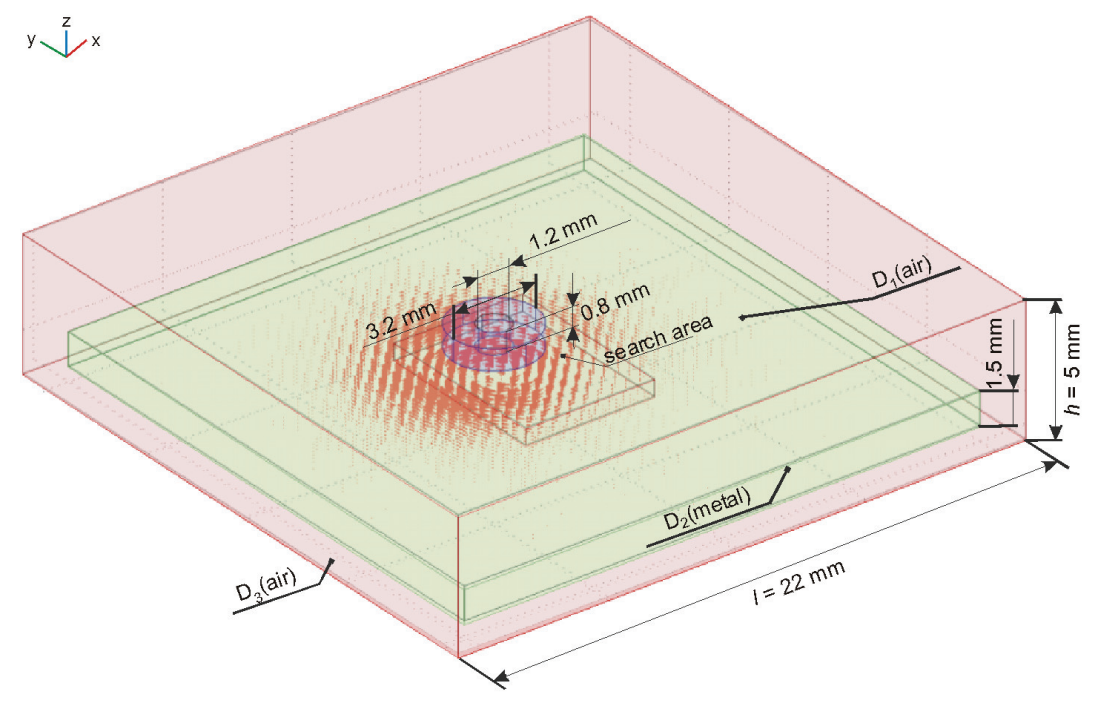

Fig. 1. View of the probe-coil and the conductive plate with the region of interest

The main concept of the ECT method relies on the introduction of low frequency time-harmonic electromagnetic field in the conductive media, and on the processing of the measured signal in order to conclude about the structure of the object under study. Since this signal contains the needed information on the discontinuity, the impedance or voltage of the probe-coil can be applied to reconstruct the size of a flaw and its position.

The configuration of the simplified ECT system is shown in Figure 1. In general, the evaluation of the material condition can be made based on measured signals generated by an eddy current probe. In the quantitative approach evaluated also in this work, the parameters of a crack, e.g. width, length and depth, can be assessed using the analysis of the field distribution in the area of interest.

Engineering optimization demands mostly the use of time-consuming forward numerical models. For example in the ECT technique, three major groups of numerical methods are commonly used for the forward simulations. The first group involves the numerical analysis such as the finite element method (FEM) e.g. [15], the finite difference method (FDM), and 
the volume integral method (VIM) [16]. Although they enable to build very accurate models, their main drawback is their expensive computational cost. To overcome this problem, one can apply integral methods like the boundary element method (BEM) for the simulation of eddy current inspections of defects having a negligible or narrow opening [17]. Especially, the socalled Bowler model is particularly interesting from the Space Mapping (SM) optimization viewpoint e.g. [18], as it enables to find the numerical approximations of narrow and arbitrary narrow-shaped cracks in a numerically efficient way. The analytical models comprise a second important group. Unfortunately, they are generally based on symmetry assumptions in the considered models and therefore they can be applied only in 2D cases e.g. [19]. Thus, these methods cannot be used for the solution of the 3D eddy current inverse problem that is considered here. The last group of techniques are based on artificial neural networks or fuzzy logic techniques $[8,9]$, and are therefore very fast. Nevertheless, their application is rather limited to the area in parameter space, for which the model has been trained.

The availability of the forward models, based on the FEM simulation as well as integral methods for inspections of narrow cracks, allows us to apply the space mapping optimization to defect recognition arising in the ECT type NDT. Therefore, in this work, FEM simulations are used as a fine model, while the reduced VIM approach for 3D flaws has been used as a coarse model after introducing some simplifications. This speeds up the calculation and still results in appropriate numerical approximations of the electromagnetic field [3].

\subsection{Model of the considered test problem}

In the present work we investigate a simplified model of a nondestructive testing system, which is a variant of the simplified model of the ECT system analyzed in the JSAEM benchmark problem 2 [14]. This model, shown in Fig. 1, consists of a pancake coil, located above a flat plate with a surface crack. For the inverse problem, we focus on a limited area of the conductive plate, the so-called search area. Its size, as well as the size of the defects under consideration, is based on the study examples analyzed in [3] and [28]. Thus, in our work we consider the model of arrangement that consists of air domains $D_{1}$ and $D_{3}\left(\varepsilon_{0}=8.854 \times 10^{-12} \mathrm{Fm}^{-1}, \mu_{0}=\right.$ $\left.=4 \pi \times 10^{-7} \mathrm{Hm}^{-1}\right)$ and the region $D_{2}\left(\varepsilon_{0}, \mu_{0}, \sigma_{0}=0.98 \times 10^{6} \mathrm{Sm}^{-1}\right)$, that is a plate consisting of the conductive metal INCONEL 600. In the restricted area of the last region, it is assumed that the 3D slot with a conductivity $\sigma(\mathbf{r})(\mathbf{r}=(x, y, z))$, bounded by a domain $\Omega$, is placed in the conductive plate. In the presented numerical tests, the following types of defects are considered: half ellipsoid shaped, cylinder shaped and rectangular parallelepiped shaped cracks, that are reconstructed using the Space Mapping based inversion procedure. The source of the field, located in the region $D_{1}$, is a 140 turn axis-symmetric shape type of the coil with internal and external diameters of 1.2 and $3.2 \mathrm{~mm}$ respectively, and has a thickness of $0.8 \mathrm{~mm}$. Additionally, in the considered model, this probe-coil is asymmetrically placed $x_{\mathrm{c} 0}=1 \mathrm{~mm}$, $y_{\mathrm{c} 0}=0 \mathrm{~mm}$ in order to guarantee a suitable covering of the interest region. The exciting coil is driven by a sinusoidal varying current with frequency $f=100 \mathrm{kHz}$ (skin depth $\delta=1.5 \mathrm{~mm}$ ). In the ECT non-destructive method, the solution of the forward problem allows to determine the probe impedance variation. In the test under consideration, the probe impedance is calculated at $N=N_{\mathrm{X}} \times N_{\mathrm{Y}}=7 \times 7=49$ coil positions, with a lift-off parameter of $0.5 \mathrm{~mm}$. The scanning 
points during the simulation are as follows: $x$ has been changed from -0.3 to $0.3 \mathrm{~mm}$ with step $0.1 \mathrm{~mm}$, while $y$ ranged from -0.75 to $0.75 \mathrm{~mm}$ with step $0.25 \mathrm{~mm}$. The simulation of one measurement scan using a $\mathrm{FE}$ analysis for 49 positions of a probe-coil requires about $3.26 \mathrm{~h}$ on our system ${ }^{1}$.

For the purpose of defect reconstruction, a combination of a fine model (FEM simulations) with a coarse model (a simplified VIM approach) is applied by means of the SM methodology. In this way the advantages of the two approaches can be combined in the proposed inversion algorithm.

\subsection{FE analysis as a fine forward model}

In the proposed approach, a finite element (FE) model is constructed in order to obtain the accurate solution of the ECT problem. The 3D model shown in Figure 1 is based on the A- $V$ formulation, where A means the magnetic vector potential, while $V$ stands for the electric scalar potential. Thus, the 3D field distribution for a time-varying harmonic case after neglecting a displacement current and using the Coulomb gauge is governed by the following equations [20]

$$
\begin{gathered}
\nabla \times \frac{1}{\mu} \nabla \times \mathbf{A}-\nabla \frac{1}{\mu} \nabla \cdot \mathbf{A}+\sigma(j \omega \mathbf{A}+\nabla V)=0 \text { in } D_{2}, \\
\nabla \cdot \sigma(j \omega \mathbf{A}+\nabla V)=0 \text { in } D_{2}, \\
\nabla \times \frac{1}{\mu} \nabla \times \mathbf{A}=\mathbf{J}^{s} \text { in } D_{1,3},
\end{gathered}
$$

where considered regions $D_{1,3}$ and $D_{2}$ stand for the surrounding free space and the eddy current domain, while $\sigma, \mu \omega$ mean the permeability and conductivity of the media and the angular frequency of density current excitation $\mathbf{J}^{\mathrm{s}}$, respectively. The model of the ECT set up after providing the spatial discretization with tetrahedral finite elements is presented in Figure 2.

After expanding the $\mathbf{A}, V$ potentials in terms of shape function according to the Galerkin technique and imposing a proper boundary condition, the solution of the forward problem defined by equations (1)-(3) takes the form of a system of algebraic equations. This equation system may be solved by using either a direct or an iterative method. In our case, the GMRES solver was applied for this purpose.

\subsection{Integral formulation for an eddy current specialization}

In contrast to the above-mentioned FE analysis, an integral formulation is applied where only the so-called search domain of the plate is divided into a regular grid of cubes. Moreover, we assign to each volumetric element of the conductive material an uniform value of the

\footnotetext{
${ }^{1}$ The simulations are conducted on a 64 bit platform that consists of 2 dual core Intel Xuon of $2.0 \mathrm{GHz}$ with 32 Gb RAM memory.
} 
electrical conductivity. Therefore, in such approach the overall conductivity profile of the search domain consists of a piecewise constant distribution of real values with some discontinuities that correspond with the cracks.

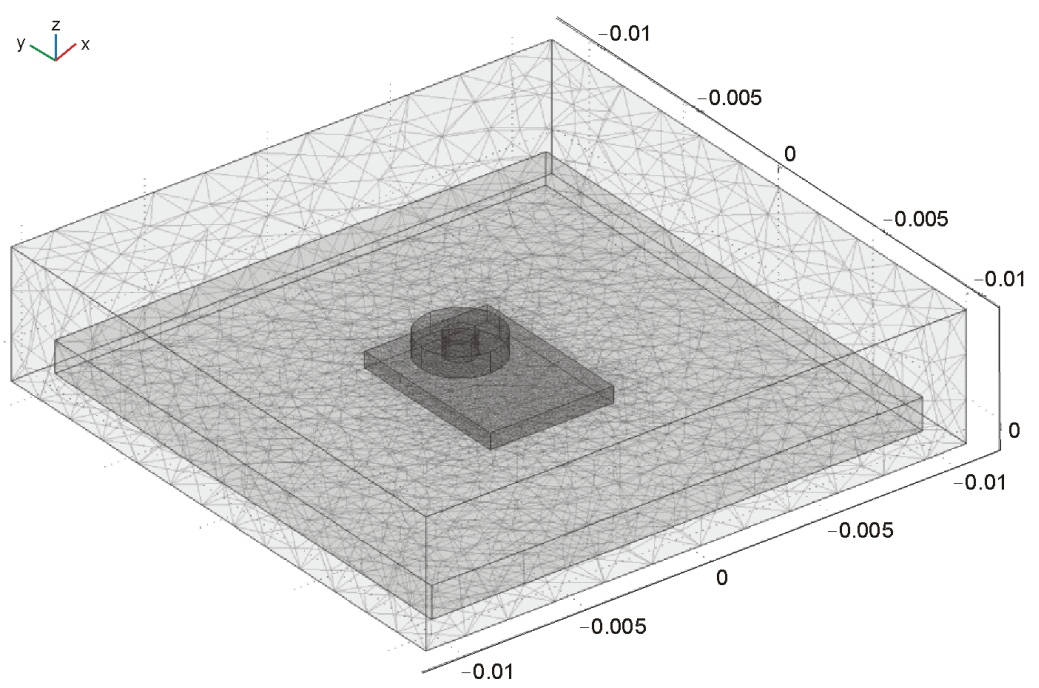

Fig. 2. 3D finite element mesh of ECT system
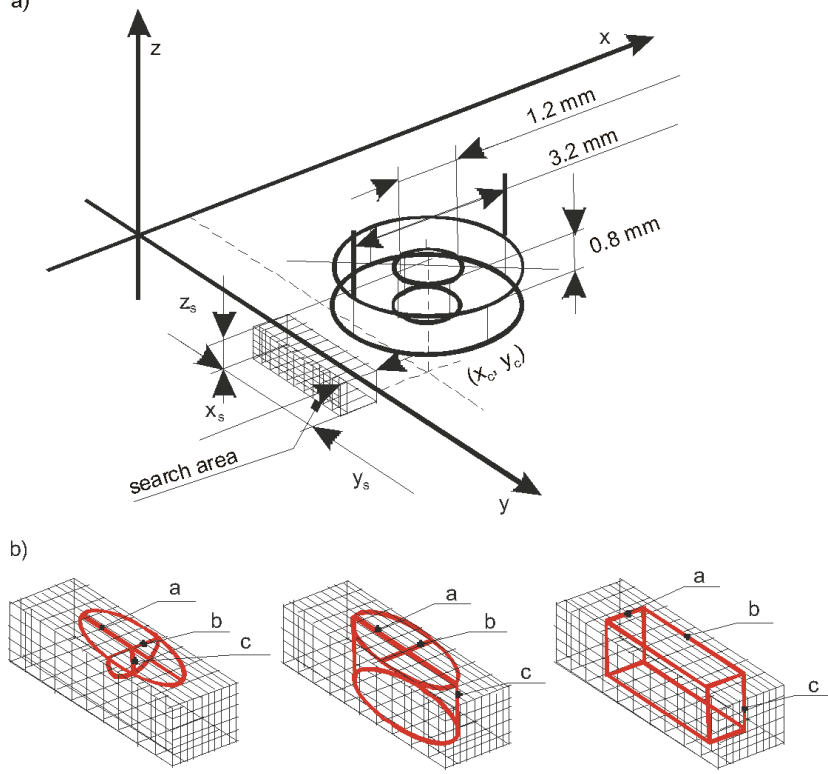

Fig. 3. Model applied in the integral formulation: a) View of probe-coil with a gridded non-conductive surface slot, b) the type of considered defects: half ellipsoid shaped, cylinder shaped and rectangular parallelepiped shaped 
Let us now consider a three-layer stratified medium that is located in the 3D Cartesian coordinate system, shown in Figure 3. Taking into account that only the linear, isotropic and non-magnetic media are investigated, the configuration of the analyzed model consists of the air regions $D_{1}$ and $D_{3}\left(\varepsilon_{0}\right.$ and $\left.\mu_{0}\right)$, and $D_{2}$ being a conductive plate made of Inconel 600 $\left(\varepsilon_{0}, \mu_{0}, \sigma_{0}\right)$, in which the $3 \mathrm{D}$ bounded slot with conductivity $\sigma(\mathbf{r})$ is placed. As a consequence, the different media are characterized by their propagation constants $k_{i}$,

$$
k^{2}(\mathbf{r})=\left\{\begin{array}{l}
k_{1}^{2}=k_{3}^{2}=\omega^{2} \mu_{0} \varepsilon_{0} \quad \text { when } \mathbf{r} \in D_{1}, D_{3} \\
k_{2}^{2} \approx j \omega \mu_{0} \sigma_{0} \quad \text { when } \mathbf{r} \in D_{2},
\end{array}\right.
$$

where $i$ is changing in the range of 1 to 3 dependent on the described regions.

Furthermore, we assume that the volumetric defect $V_{f}$ with the finite search domain specified by width $x_{\mathrm{s}}$, length $y_{\mathrm{s}}$, and deepness $z_{\mathrm{s}}$ can be described at any point $\mathbf{r}$ by means of the socalled contrast function

$$
\chi(\mathbf{r})=\frac{\sigma(\mathbf{r})-\sigma_{0}}{\sigma_{0}}= \begin{cases}-1 & \text { when } \mathbf{r} \in V_{f} \\ 0 & \text { otherwise },\end{cases}
$$

Thus, the distribution of eddy currents, induced in the plate due to the excitation coil in the presence of defect in the model, can be expressed by a Fredholm second-kind vector integral equation. The application of the Green's theorem and taking into account the boundary conditions at material discontinuities as well as the radiation condition at infinity, allows to define the associated electric field distribution as the integral equation e.g. [21]

$$
\mathbf{E}(\mathbf{r})=\mathbf{E}^{(i)}(\mathbf{r})+k_{2}^{2} \int_{D_{2}} \mathbf{G}\left(\mathbf{r} \mid \mathbf{r}_{0}\right) \chi\left(\mathbf{r}_{0}\right) \mathbf{E}\left(\mathbf{r}_{0}\right) d \mathbf{r}_{0} \quad \mathbf{r}, \mathbf{r}_{0} \in D_{2} .
$$

Here, $\mathbf{E}^{(i)}(\mathbf{r})$ means the incident field caused by the primary source, when the defect is absent in the model, while $\mathbf{E}(\mathbf{r})$ is the total electric field. The last term, $\mathbf{G}\left(\mathbf{r} \mid \mathbf{r}_{0}\right)$ is the dyadic electric-electric Green's function (both source and field observation in $D_{2}$ ), which satisfies the dyadic Helmholtz equation [22]

$$
\nabla \times \nabla \times \mathbf{G}\left(\mathbf{r} \mid \mathbf{r}_{0}\right)-k_{2}^{2} \mathbf{G}\left(\mathbf{r} \mid \mathbf{r}_{0}\right)=\mathbf{I} \delta\left(\mathbf{r}-\mathbf{r}_{0}\right),
$$

with $\mathbf{I}$ the unit dyad, $\delta$ the Dirac impulse that here represents a unit point current source with orientation along the three coordinate axes. Moreover, for the eddy current problem, the following reciprocity relationship $\mathbf{G}\left(\mathbf{r} \mid \mathbf{r}_{0}\right)=\mathbf{G}\left(\mathbf{r} \mid \mathbf{r}_{0}\right)^{T}$ is satisfied, where $T$ means transposition operator [20]. After multiplying equation (6) by $\sigma_{0} \chi(\mathrm{r})$ and defining

$$
\mathbf{P}^{(i)}(\mathbf{r})=\chi(\mathbf{r}) \mathbf{J}^{(i)}(\mathbf{r})
$$

as the incident eddy current sources associated to the primary field, which is set to null except for $V_{f}$, the eddy current phenomenon inside the volumetric defect is described by 


$$
\mathbf{P}(\mathbf{r})=\mathbf{P}^{(i)}(\mathbf{r})+k_{2}^{2} \int_{D_{2}} \mathbf{G}\left(\mathbf{r} \mid \mathbf{r}_{0}\right) \chi\left(\mathbf{r}_{0}\right) \mathbf{P}\left(\mathbf{r}_{0}\right) d \mathbf{r}_{0} \quad \mathbf{r}, \mathbf{r}_{0} \in D_{2}
$$

The incident eddy current density in the center of the volumetric element of a breaking slot can then be computed using Dodd and Deeds approach [19] or the numerically very effective Truncated Region Eigenfunction Expansion (TREE) method introduced by Theodoulidis [23]. Since the $\mathbf{P}(\mathbf{r})$ is known (by solving numerically eq. 9), the variation of the impedance after using the reciprocity theorem relating the scattered field at the coil $\mathbf{E}^{(\mathrm{s})}(\mathbf{r})$ and incident field at the flaw $\mathbf{E}^{(\mathrm{i})}(\mathbf{r})$, is given by [19]:

$$
\Delta Z=-\frac{1}{I^{2} \sigma_{0}} \int_{D_{2}} \mathbf{J}^{(i)}(\mathbf{r}) \mathbf{P}(\mathbf{r}) d \mathbf{r} \quad \mathbf{r} \in V_{f},
$$

where $I$ means the magnitude of the excitation current. The integration is conducted over the volume of the flaw and $\mathbf{P}(\mathbf{r})$ may be interpreted as the effective current dipole density at the slot resulting from the change in conductivity between the host and the flaw. Equation (9) with the unique constraint of vanishing normal component of the eddy current density $P_{\mathrm{n}}(\mathbf{r})=0$ on the crack surface, after discretization of the defect volume with a regular cubical grid of $N$ elements and the application of the point matching procedure, is then transformed to linear systems of equations

$$
-k_{2}^{2}[\mathbf{G}][\mathbf{P}]=\left[\mathbf{J}_{0}\right] .
$$

Here, $\mathbf{J}_{0}$ means a vector of incident eddy current density, $\mathbf{G}$ is a square matrix with the Green's functions elements [33], and $\mathbf{P}$ stands for a vector with the unknown dipole density for each element of the discretized defect. Certainly, the solution of the vector integral equation (9) has this advantage that it accounts for all the phenomena in the defect area. However, on the other hand, such approach demands a high computational load. Therefore, we decided to derive the coarse model not from the full vector equation (10). For this purpose, the reduced VIM approach for a scalar, yet 3D equation, is used.

\subsection{Coarse model as the reduced to the only one component VIM model}

This approach is analogous to the investigation presented by the authors in $[24,25]$. According to their research, accounting for only one component of electric fields, in our case the $x x$ component of the dyadic Green function, as well as only the $x$ component of eddy current density, leads to a good numerical approximation of an electric field. This kind of simplification implies the reduction of the vector integral equation (9) to a scalar 3D version. For the analysis of the ECT model in the Cartesian coordinate system, we propose to apply an analogues approach. Thus, when the unique constraint of vanishing normal component of the eddy current density on the crack surface, in our case $J_{x}(\mathbf{r})$, is defined as follows $[21,22]$

$$
J_{x}(\mathbf{r})=0,
$$

the linear system of equations (11) can be written as 


$$
\left[\begin{array}{c}
P_{x} \\
P_{y} \\
P_{z}
\end{array}\right]=-\frac{1}{k_{2}^{2}}\left[\begin{array}{ccc}
T_{x x} & T_{x y} & T_{x z} \\
T_{y x} & T_{y y} & T_{y z} \\
T_{z x} & T_{z y} & T_{z z}
\end{array}\right]\left[\begin{array}{c}
J_{0 x} \\
0 \\
0
\end{array}\right]
$$

with inverse matrix $\mathbf{T}=\mathbf{G}^{-1}$. Since the Green dyad is diagonally dominant, the matrix of system equations $\mathbf{G}$ and the inverse matrix $\mathbf{T}$ should be diagonally dominant, too. Therefore, the other dominant terms like $T_{\mathrm{yy}}$ and $T_{\mathrm{zz}}$ can be neglected due to $J_{0 \mathrm{y}}=J_{0 \mathrm{z}}=0$, which implies that $P_{\mathrm{x}} \gg P_{\mathrm{y}}$ and $P_{\mathrm{z}}$ since $T_{\mathrm{xx}}$ is also dominant over $T_{\mathrm{xy}}$ and $T_{\mathrm{xz}}$. The justification for such approach is e.g. the inspection of steam generator tubes, where usually the cracks are very thin and long. This is the reason why the orientation of the defect can be first discovered based on measurement data. Thus, finally the equation (9) can be further reduced to its only $x$ component version which can be analyzed in a $3 \mathrm{D}$ model

$$
P_{x}^{(i)}(\mathbf{r})=-\chi(\mathbf{r}) k_{2}^{2} \int_{D_{2}} G_{22 x x}\left(\mathbf{r} \mid \mathbf{r}_{0}\right) P_{x}\left(\mathbf{r}_{0}\right) d \mathbf{r}_{0} \quad \mathbf{r} \in D_{2} .
$$

In short, after the discretization of the defect volume and the application of the point matching procedure, the eddy current phenomenon in the coarse model is described by equation (13). In this way a coarse model has been created on the basis of the fully integral formulation defined by equation (9), where some time-consuming sub-procedures related to the electric field computation were not included. To reach the convergence in the field computation, the same criterion for a coarse model as in [24] is applied. Thus, the size of the voxels is set to approximately $(\delta / 7)^{3}$, where $\delta$ is the standard skin depth. Finally, for the purpose of the inversion procedure for $3 \mathrm{D}$ flaws that demands at least $2 \mathrm{D}$ measurements or synthetic data, the impedance variation of the probe-coil for different $x_{\mathrm{c}}$ and $y_{\mathrm{c}}$ positions and given the value of a lift-off parameter is calculated using equation (10).

\subsection{Inversion procedure in a coarse model}

The main advantage of such defined coarse model, especially when used for the solution of the eddy current inverse problem, is the ability to identify any number of defects, which can be described by means of the same grid, on the basis of the pre-computed data. Thus, the inverse problem can be defined as follows. When assuming that the crack can be specified by the following set of parameters

$$
\mathbf{p}=\left\{p_{1}, p_{2}, \ldots, p_{p}\right\}
$$

and the cost function is given by

$$
Q(\mathbf{p})=\frac{1}{2} \sum_{j}\left(\Delta Z(\mathbf{p})_{j}-\Delta Z_{j}^{0}\right)\left(\Delta Z(\mathbf{p})_{j}-\Delta Z_{j}^{0}\right)^{*},
$$

then the process of defect identification can be conducted by the minimization of such defined last-square functional of the data-model. Here, $\Delta \mathrm{Z}(\mathbf{p})_{j}$ denotes the $j$-th component of the impedance change that is simulated in the coarse model and $\Delta \mathrm{Z}_{j}^{0}$ denotes the target impedance variation measured for the $j$-th probe-coil position. 
The application of the Gauss-Newton (G-N) algorithm with Tikhonov regularization for defects reconstruction requires first the calculation of the gradient of the cost function (15). However, the crucial component of the gradient is the sensitivity information. Therefore, the numerically efficient adjoint Tellegen method $[26,27]$ is used for this purpose. After assuming some parameterization of the flaw function $\chi(\mathbf{r})$ using

$$
f(\mathbf{p}, \mathbf{r})=\sum_{i=1}^{N} p_{i} \varphi_{i}(\boldsymbol{r})
$$

where $\varphi_{i}, i=1, \ldots . N$ represent a set of linearly independent basis function, while $p_{i}$ are expansion coefficients, the sensitivity formula is defined as

$$
s_{j i}=\frac{\partial Z_{j}}{\partial p_{i}}=-\sigma_{0} \int_{V^{e}} \tilde{\mathbf{E}}(\mathbf{r}) \mathbf{E}(\mathbf{r}) \frac{\partial f(\mathbf{p}, \mathbf{r})}{\partial p_{i}} d \mathbf{r} .
$$

Here, $\mathbf{E}(\mathbf{r})$ means the electric field when the flaw is absent, while $\tilde{\mathbf{E}}(\mathbf{r})$ refers to the adjoint field. Additionally, the integration is taken over the volume of the $e$-th flaw voxel. Moreover, when the impedance magnitude is considered, the sensitivity can be computed using

$$
\tilde{s}_{j i}=\frac{\operatorname{Re}\left(\Delta Z_{j}\right) \operatorname{Re}\left(s_{j i}\right)+\operatorname{Im}\left(\Delta Z_{j}\right) \operatorname{Im}\left(s_{j i}\right)}{\left|\Delta Z_{j}\right|} .
$$

It is worth noting that the gradient of the cost function can be efficiently calculated on the basis of equation (16) and/or (17) when using pre-computed data. However, due to fact that the reconstruction of the defects parameters is seriously hampered by the inherently ill-posed and non-linear nature of the eddy current inverse problem, a regularization technique such as the Tikhonov method with the Generalized Cross Validation GCV $(\lambda)$ needs to be applied [28]. The result for the application of the described inversion procedure for the identification of the cubic-like shape surface defect is shown in Figure 4.

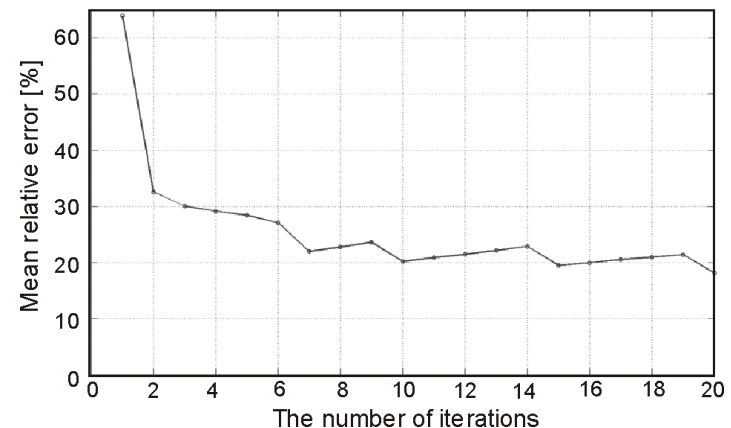

Fig. 4. The convergence history for the reconstruction of a cuboidal crack when using the coarse model based on the regularized G-N algorithm

In the numerical example, the synthetic data are obtained by solving equations (13) and (10) corresponding to the forward coarse model for the crack dimensions as follows: width 
$a=0.6 \mathrm{~mm}$, length $b=2.0 \mathrm{~mm}$ and depth $c=1.0 \mathrm{~mm}$. The search region consists of $N_{\mathrm{D}}=N_{\mathrm{X}} \times$ $N_{\mathrm{Y}} \times N_{\mathrm{Z}}=8 \times 10 \times 5=400$ voxels, where each cell of the search domain $V_{f}$ has a size of $\delta_{\mathrm{X}} \times$ $\delta_{\mathrm{Y}} \times \delta_{\mathrm{Z}}=0.1 \times 0.25 \times 0.125 \mathrm{~mm}$. Furthermore, the probe-coil is fed by a time-harmonic excitation current with frequency $f=100 \mathrm{kHz}$. Although the shape of the crack is reconstructed after 16 iterations and is computationally fast (on average only a few minutes), the obtained solution is not acceptable from the accuracy viewpoint. The level of the relative mean identification error when using this model is about $15-20 \%$. Therefore, the Space Mapping optimization needs to be applied for further improving the accuracy of defect identification.

\section{Two-level optimization method}

In the former sections, on the one hand the accurate and time-consuming numerical method were presented to solve the considered ECT forward problem. On the other hand, the much faster, but less accurate scheme based on the reduced VIM approach was shown. In order to speed up the inversion procedure, a Space Mapping is used, which enables to combine both the fine and coarse models to come to a fast and accurate algorithm for defect reconstruction.

\subsection{Introduction}

Space Mapping (SM) is a highly recognized, efficient optimization method that has found a broad application to solve a wide range of engineering problems arising in various industry fields [10-13, 29-31]. The main concept behind SM is to replace the traditional direct optimization procedure based on the accurate analysis of a computationally slow fine model with an iterative optimization and updating of a coarse model, which therefore, is cheaper to evaluate but also less precise. In consequence, the coarse model is used for an exploration while the fine model is considered only for a limited number of times (i.e. exploitation). An example of a fine simulation might be a model of a device analyzed in an electromagnetic simulator or a numerical model of a non-destructive system such as ECT, MFL. According to the SM methodology, an analytical formula describing the behaviour of the device, a circuit simulation of this device or a simplified numerical scheme after reduction of time-demanding subroutines would serve as a coarse model. Assuming that the misalignment between both models can be minimized or is just not significant, the SM optimization allows for an essential reduction of the CPU time needed for obtaining reliable results typically after only a few iterations, this in contrast to direct optimization procedures [10].

In many practical applications in the engineering field the goal is to solve

$$
\mathbf{x}_{f}^{*}=\arg \min _{\mathbf{x}_{f} \in X_{f}} \Phi\left(\mathbf{R}_{f}(\mathbf{x})\right)^{2},
$$

where

$$
\arg \min _{\mathbf{x} \in X} f(\mathbf{x})=\{\mathbf{x} \mid \forall \mathbf{y}: f(\mathbf{y}) \geq f(\mathbf{x})\} \text { and where } f \in R .
$$


Here, $\mathbf{R}_{f}: X_{f} \rightarrow R^{m}$ denotes the response vector of the fine model, while a functional $\Phi$ (cost function) can be defined by e.g. a second norm

$$
\Phi\left(\mathbf{R}_{f}(\mathbf{x})\right)=\left\|\mathbf{R}_{f}(\mathbf{x})-\mathbf{R}_{f}^{0}(\mathbf{x})\right\|,
$$

where $\mathbf{R}_{f}^{0}(\mathbf{x})=\left[\mathbf{R}_{f .1}^{0}, \mathbf{R}_{f .2}^{0}, \ldots, \mathbf{R}_{f . M}^{0}\right]$ means the target response.

Instead of solving problem (18) using direct optimization of the time-consuming fine model, we consider surrogate models which are a good local approximation of the fine model. It is also assumed that they are computationally non-expensive and therefore suitable for an iterative optimization. From these reasons, we investigate an optimization algorithm that produces a sequence of results $\mathbf{x}_{\mathbf{f}}^{(i)}(i=1,2, \ldots, K)$

$$
\mathbf{x}_{f}^{(i)}=\arg \min _{\mathbf{x}} \Phi\left(\mathbf{R}_{S}^{(i)}(\mathbf{x})\right),
$$

while $\mathbf{R}_{S}^{(i)}(\mathbf{x})$ denotes a family of surrogate models. However, the surrogates are created on the basis of a coarse model and an auxiliary mapping defined during the so-called parameter extraction process by means of

$$
\mathbf{p}^{(\mathrm{i}+1)}=\arg \min _{p}\left\|\mathbf{R}_{f}\left(\mathbf{x}^{(\mathrm{i})}\right)-\overline{\mathbf{R}}_{S}\left(\mathbf{x}^{(\mathrm{i})}, \mathbf{p}^{(i)}\right)\right\| .
$$

Here, $\mathbf{R}_{\mathrm{S}}^{(i)}(\mathbf{x})=\overline{\mathbf{R}}_{S}\left(\mathbf{x}, \mathbf{p}^{(i)}\right)$ is a generic SM surrogate model, that is a coarse model $\boldsymbol{R}_{c}$ with a typically linear transformation, where $\boldsymbol{R}_{c}: X_{f} \rightarrow R^{m}[10]$.

Different types of surrogate models have been presented in the literature during the last decade $[10,13]$, e.g.:

- models that use typically a linear transformation in the parameter space e.g. Aggressive Space mapping (ASM), or an input SM [10, 11],

- models based on the transformation in the response parameter space, for instance, an output SM [13] or the Manifold Mapping optimization [29],

- models that exploit the parameter and response spaces in order to align the surrogate with a fine model, e.g. the Response and Parameter Mapping [12],

- the Implicit Space Mapping [32] which allows the separation of the parameters and design variables used in the process of alignment the surrogate with a fine model,

- the custom models that exploit the parameters which are characteristic for a given design problem [10].

Summarizing, the flow of a SM-based algorithm can be written as [31]

- step 1) set $i=1$ and choose the initial solution $\mathbf{x}^{(l)}$ for the given fine model and coarse model,

- step 2) calculate the fine model in order to find $\mathbf{R}_{f}\left(\mathbf{x}^{(i)}\right)$,

- step 3) evaluate in the surrogate model $\mathbf{R}_{\mathbf{s}}^{(i)}$ using (21),

- step 4) based on $\mathbf{x}^{(i)}$ and $\mathbf{R}_{\mathbf{s}}{ }^{(i)}$ determine $\mathbf{x}^{(i)}$ using (20),

- step 5) if the stop condition is not fulfilled, choose step 2, otherwise finish the calculation.

In this paper, two types of two-level techniques are considered for solving the eddy current inverse problem: the ASM method and the MM algorithm. Assuming the $j$-dimensional vector of the probe impedance in case of the coarse (fine) model for certain $i$-dimensional flaw para- 
meters vector $\mathbf{x}_{c} \in X_{c}\left(\mathbf{x}_{f} \in X_{f}\right)$ is denoted by $\mathbf{c}\left(\mathbf{x}_{c}\right) \in \Omega_{\mathrm{c}}\left(\mathbf{f}\left(\mathbf{x}_{f}\right) \in \Omega_{f}\right)$, the optimization problem (18) can be reformulated and shown in an explicit form as

$$
\mathbf{x}_{f}^{*}=\arg \min _{\mathbf{x}_{f} \in X_{f}}\left\|\mathbf{f}\left(\mathbf{x}_{f}\right)-\mathbf{y}\right\|
$$

where $\mathbf{y}$ means the target impedance variation, which is obtained by either simulation or as a result of the conducted measurements.

\subsection{Aggressive space mapping}

In the space mapping methods, a coarse model is used for generating surrogates that need to approximate and exploit a fine model. Therefore, the mapping between parameter spaces of both models is constructed, so that

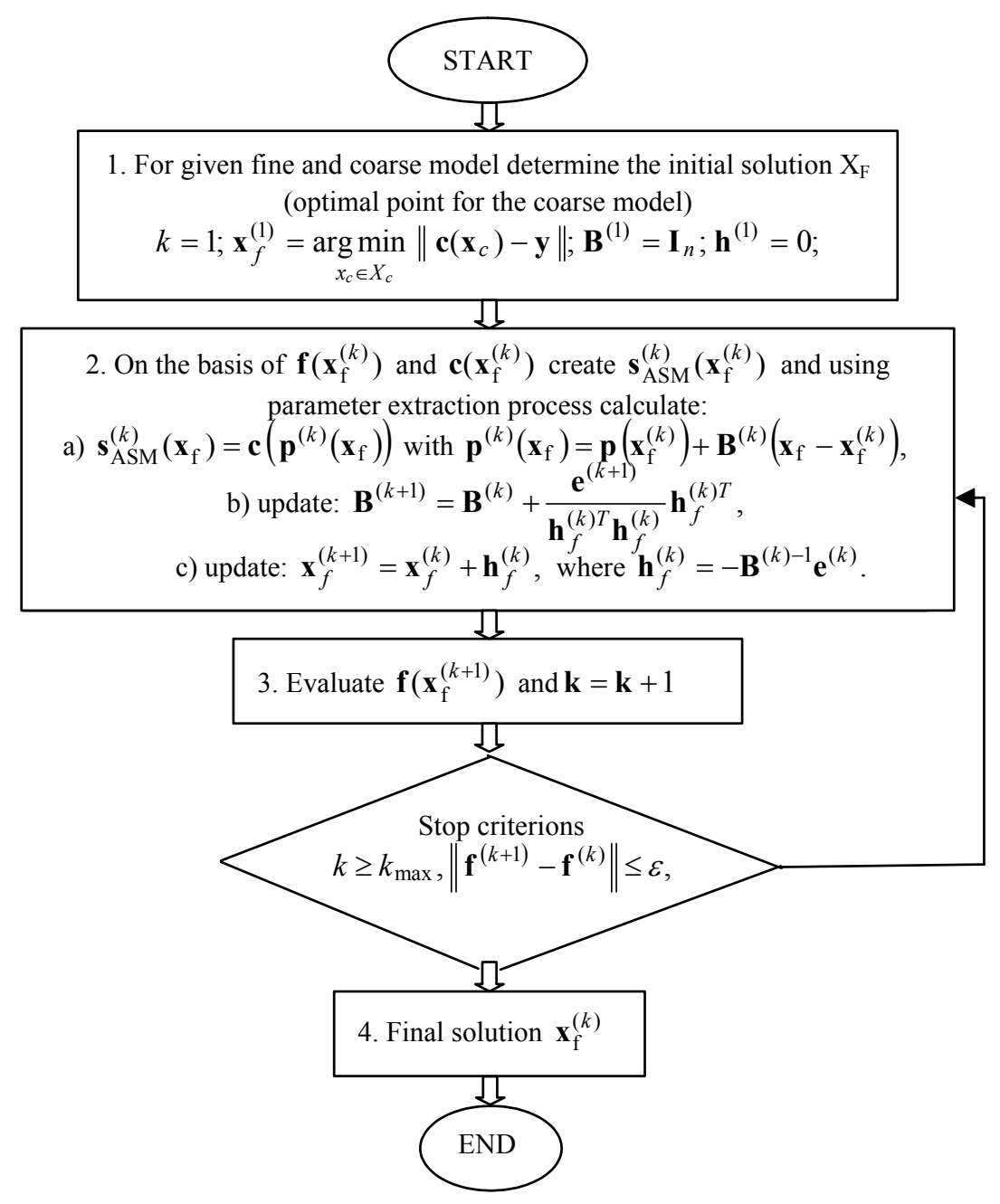

Fig. 5. Flowchart of inversion procedure based on ASM algorithm 


$$
\mathbf{f}\left(\mathbf{x}_{f}\right) \approx \mathbf{c}\left(\mathbf{p}\left(\mathbf{x}_{f}\right)\right)
$$

can be satisfied.

Hence, in this case the suitable surrogates are found as a result of the so-called parameter extraction process $(\mathrm{PE})$ that is provided in such a way that the coarse model matches the fine model.

In the aggressive type of the SM method (Fig. 5), the surrogate model in the $k$-th iteration is given by [10]

$$
\mathbf{s}_{\mathrm{ASM}}^{(k)}\left(\mathbf{x}_{f}\right)=\mathbf{c}\left(\mathbf{p}^{(k)}\left(\mathbf{x}_{f}\right)\right),
$$

while the next result $\mathbf{x}_{\mathrm{f}}^{(k)}$ can be computed using

$$
\mathbf{x}_{f}^{(k)}=\arg \min _{\mathbf{x}_{f} \in X_{c}}\left\|\mathbf{s}_{\mathrm{ASM}}^{(k)}\left(\mathbf{x}_{f}\right)-\mathbf{y}\right\|,
$$

with a mapping function defined as

$$
\mathbf{p}^{(k)}\left(\mathbf{x}_{f}\right)=\mathbf{p}\left(\mathbf{x}_{f}^{(k)}\right)+\mathbf{B}^{(k)}\left(\mathbf{x}_{f}-\mathbf{x}_{f}^{(k)}\right)
$$

Here, $\mathbf{x}_{f}^{(k)}$ is the $k$-th quasi Newton iteration with $\mathbf{B}^{(k)}$ being an approximation of the $\mathbf{p}\left(\boldsymbol{x}_{f}\right)$ Jacobian that can be updated using Broyden's rank formula [10]. Thus, in this way the direct optimization of the fine model is replaced by the iterative optimization of the cheaper to calculate but less accurate surrogate models based on the coarse model.

However, the inversion procedure based on the ASM techniques might fail if there is a significant misalignment between both models responses. In such case, the solution of optimization problem defined by (25) does not necessarily converge to the local or global minimum.

\subsection{Manifold mapping}

In contrast to the SM approach where mapping is performed in the parameter space, the MM algorithm (Fig. 6) employs an affine mapping only between the responses of the coarse and fine models. Thus, in order to define an affine map between two vector spaces, this technique applies a linear transformation followed by a translation [29]. In this way it performs the response correction by establishing a surrogate model with an affine mapping in the response spaces of both considered models.

The MM algorithm uses the following type of surrogate model

$$
\mathbf{s}_{\mathrm{MM}}^{(k)}\left(\mathbf{x}_{f}\right)=\mathbf{f}\left(\mathbf{x}_{f}^{(k)}\right)+\mathbf{D}^{(k)}\left(\mathbf{c}\left(\mathbf{x}_{f}\right)-\mathbf{c}\left(\mathbf{x}_{f}^{(k)}\right)\right)
$$

where $\mathbf{D}^{(k)}$ is a regular, so-called rotation matrix, and $\mathbf{f}\left(\mathbf{x}_{f}^{(k)}\right)$ plays a role of a translation vector. Under the assumption that $\mathbf{x}_{\mathrm{c}}=\mathbf{x}_{\mathrm{f}}$ the $\mathbf{x}_{f}^{(k+1)}$ is defined in the MM algorithm as

$$
\mathbf{x}_{f}^{(k)}=\arg \min _{\mathbf{x}_{f} \in X_{c}}\left\|\mathbf{s}_{\mathrm{MM}}^{(k)}\left(\mathbf{x}_{f}\right)-\mathbf{y}\right\| .
$$

It follows from (27) and (28) that, the Manifold Mapping technique can be used without a necessity of computing the exact gradient information. 


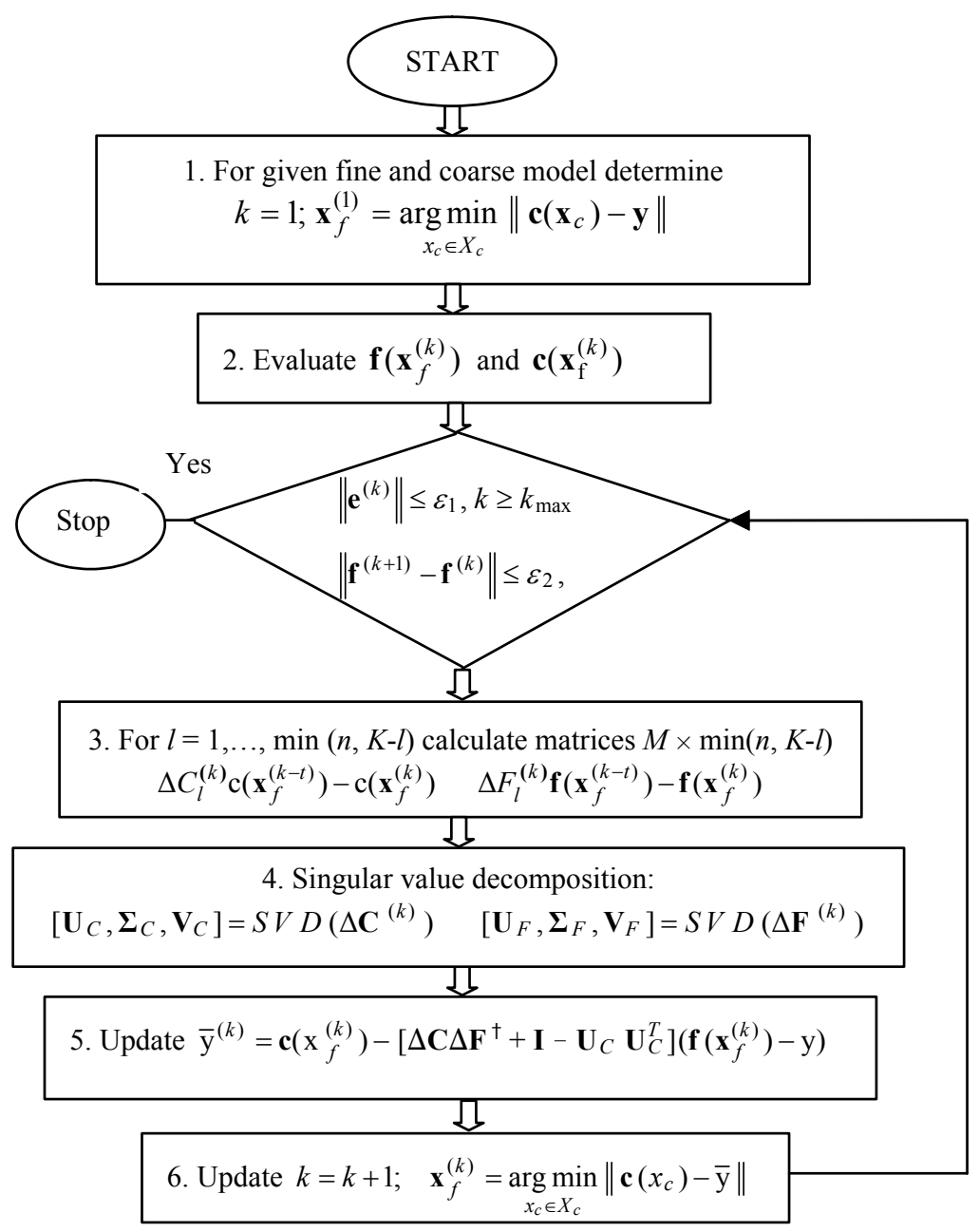

Fig. 6. Flowchart of inversion procedure based on MM optimization

\section{Result for the defects reconstruction}

In order to verify the proposed inversion technique based on SM optimization, we consider the reconstruction of three different crack shapes (Fig. 3). In the presented numerical examples, the conductivity distribution in an anomalous area of a conductive material is described by means of a known function. The parameters of the artificial cracks are given in Table 1.

Table 1. Parameters of the artificial cracks

\begin{tabular}{l|c|c|c}
\hline \multicolumn{1}{c|}{ Crack name } & a) width $[\mathrm{mm}]$ & b) length [mm] & c) depth [mm] \\
\hline Crack 1 (ellipsoidal) & 0.3 & 1.0 & 0.5 \\
\hline Crack 2 (cuboidal) & 0.6 & 2.0 & 0.5 \\
\hline Crack 3 (cylindrical) & 0.3 & 1.0 & 0.5 \\
\hline
\end{tabular}


Synthetic data is generated by solving the adequate forward problem, which is treated as the reference impedance for solving the inverse problem. The FE simulations are calculated using equations (1)-(3) and acts as fine model within the SM scheme. The ECT system under investigation is depicted in Figure 7. It consists of 94255 finite elements and the number of degree of freedom is solved for 143075. The search domain has a size of $D=\Delta X_{\mathrm{s}} \times \Delta Y_{\mathrm{s}} \times$ $\times \Delta Z_{\mathrm{s}}=0.8 \times 2.25 \times 0.625 \mathrm{~mm}$, and is placed in the plate made of Inconel 600 with dimensions $22 \times 22 \times 1.25 \mathrm{~mm}$. The size of probe as well as its localization versus the search area is shown in Figure 8. For such configuration, the probe impedance is measured at $N=N_{X} \times$ $N_{Y}=7 \times 7=49$ coil positions, with a lift-off parameter of $0.5 \mathrm{~mm}$. The simulation of one measurement scan in the FE case for 49 positions of the coil, when $x$ is changing from -0.3 to $0.3 \mathrm{~mm}$ with step $0.1 \mathrm{~mm}$ and $y$ is ranging from -0.75 to $0.75 \mathrm{~mm}$ with step $0.25 \mathrm{~mm}$, requires about $3.26 \mathrm{~h}$ on our system.

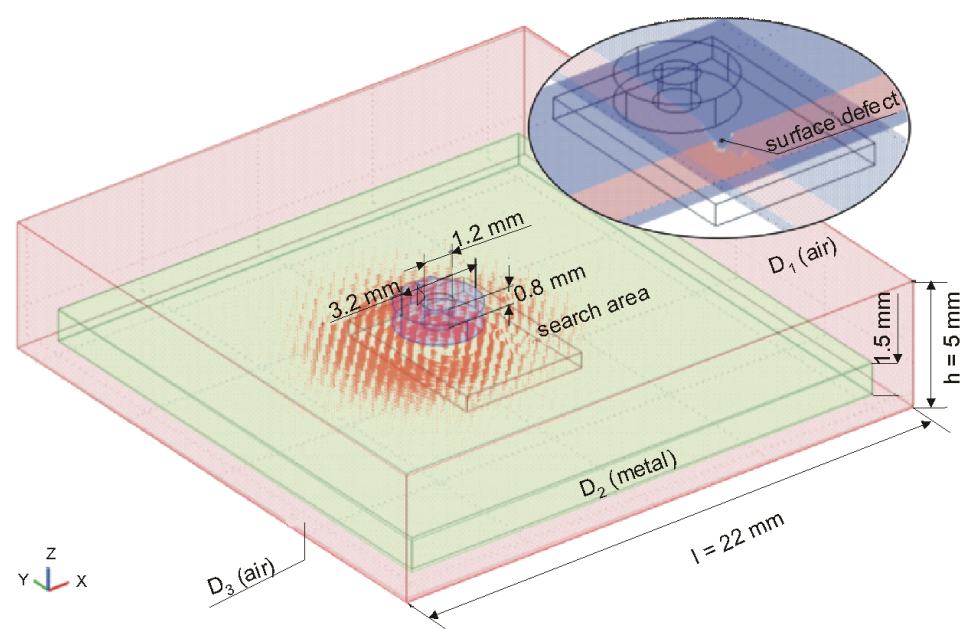

Fig. 7. View of the probe-coil, the conductive plate with the region of interest

As coarse model, the scalar 3D VIM described by equation (13) with condition (12a) is considered. In the coarse model, the test domain is divided into $N D=N_{\mathrm{X}} \times N_{\mathrm{Y}} \times N_{\mathrm{Z}}=8 \times 10 \times$ $\times 5=400$ voxels, each of size $\delta_{\mathrm{X}} \times \delta_{\mathrm{Y}} \times \delta_{\mathrm{Z}}=0.1 \times 0.25 \times 0.125 \mathrm{~mm}$. It is worth mentioning that the single-time analysis in this model for 49 positions of the probe takes about a few seconds using pre-computed data, while the coarse model solution during the inverse problem itself, also based on the pre-computed data, takes only about $5 \mathrm{~min}$. These features make this approach perfectly suitable as an efficient coarse model. The result of the simulation in both models is presented in Figure 8. For the calibration purpose, one point procedure using maximal value is used.

The two above-mentioned SM techniques are first implemented and then tested in eddy current inverse problem. 


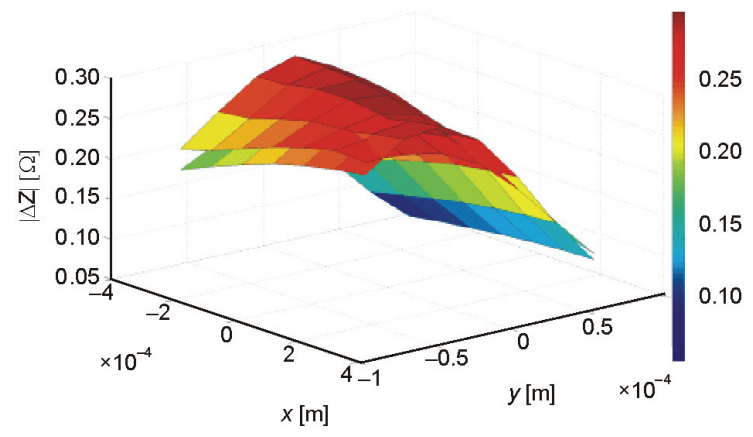

Fig. 8. The result for the calibration of the FEM and the reduced VIM model for the assumed reference crack

\subsection{Defects reconstruction based on ASM algorithm}

The result of reconstruction process based on the regularized ASM algorithm is summarized in Table 2.

Table 2. Inversion results for regularized ASM optimization of investigated crack

\begin{tabular}{c|c|c|c|c|c|c}
\hline $\begin{array}{c}\text { Name of } \\
\text { defect }\end{array}$ & $\begin{array}{c}\text { Initial } \\
\text { point } \\
{[\mathrm{mm}]}\end{array}$ & $\begin{array}{c}\text { MRE for } \\
\mathbf{x}_{c}[\%]\end{array}$ & $\begin{array}{c}\text { No of coarse } \\
\text { model } \\
\text { evaluation }\end{array}$ & $\begin{array}{c}\text { No of fine } \\
\text { model } \\
\text { evaluations }\end{array}$ & $\begin{array}{c}\text { Reconstructed } \\
\text { size of defect }\end{array}$ & $\begin{array}{c}\text { MRE for } \mathbf{x}_{f} \\
{[\%]}\end{array}$ \\
\hline $\begin{array}{c}\text { Crack 1 } \\
\text { (ellipsoidal } \\
\text { flaw) }\end{array}$ & {$\left[\begin{array}{c}0.20 \\
0.75 \\
0.375\end{array}\right]$} & $12 \%$ & 6 & 7 & {$\left[\begin{array}{l}0.23 \\
1.04 \\
0.49\end{array}\right]$} & $9.7 \%$ \\
\hline $\begin{array}{c}\text { Crack 2 } \\
\text { (cuboidal } \\
\text { flaw) }\end{array}$ & {$\left[\begin{array}{c}0.100 \\
0.375 \\
0.187\end{array}\right]$} & $14 \%$ & 5 & 6 & {$\left[\begin{array}{l}0.559 \\
1.947 \\
0.388\end{array}\right]$} & $7.2 \%$ \\
\hline $\begin{array}{c}\text { Crack 3 } \\
\text { (cylindrical } \\
\text { flaw) }\end{array}$ & {$\left[\begin{array}{c}0.100 \\
0.375 \\
0.187\end{array}\right]$} & $13 \%$ & 4 & 5 & {$\left[\begin{array}{l}0.319 \\
0.986 \\
0.408\end{array}\right]$} & $8.6 \%$ \\
\hline
\end{tabular}

Hence, the first column shows the values of starting points for the three kinds of considered cracks, the second column indicates the values of the mean relative error calculated after providing the initial reconstruction in the coarse model, while the third and fourth columns represent the number of coarse and fine model evaluations, respectively. Finally, in the last column one can see the value of the mean relative error (i.e. accuracy of inversion procedure) associated to the reconstructed parameters (with values presented in the next to last column) of defect.

The distribution of both impedances: target and that after providing the defects reconstruction is presented on Figures. 9, 10 and 11, respectively. 


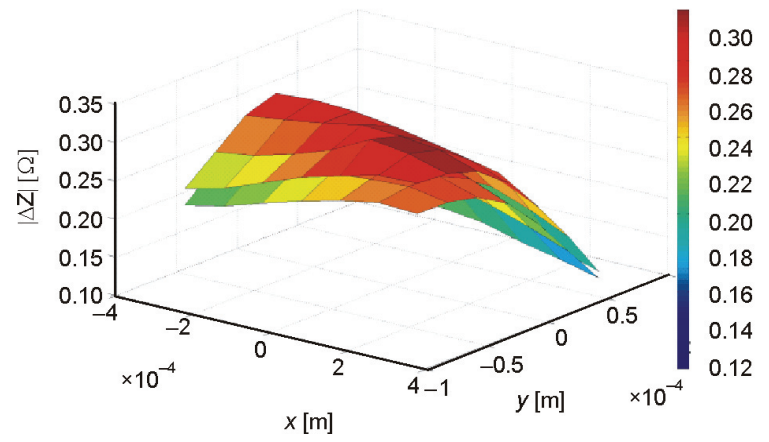

Fig. 9. Comparison of the target impedance magnitude with that obtained at the ASM optimal point in case of ellipsoidal defect (crack 1)

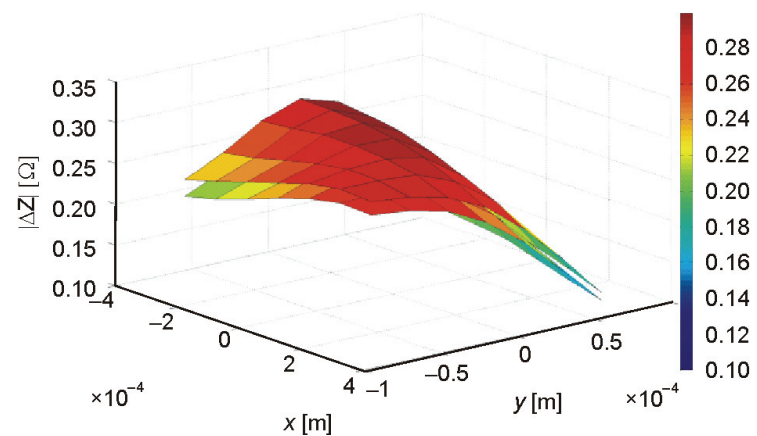

Fig. 10. Comparison of the target impedance magnitude with that obtained at the ASM optimal point in case of cylindrical defect (crack 3 )

\subsection{Defects reconstruction by MM based inversion procedure}

In Table 3, the results of the reconstruction when using the MM are presented. The table is organized in the same way as the previous one.

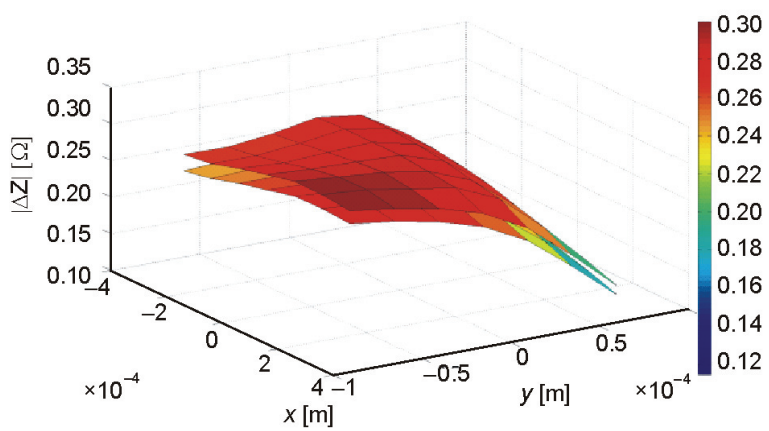

Fig. 11. Comparison of the target impedance magnitude with that obtained at the ASM optimal point in case of cubical defect (crack 2) 


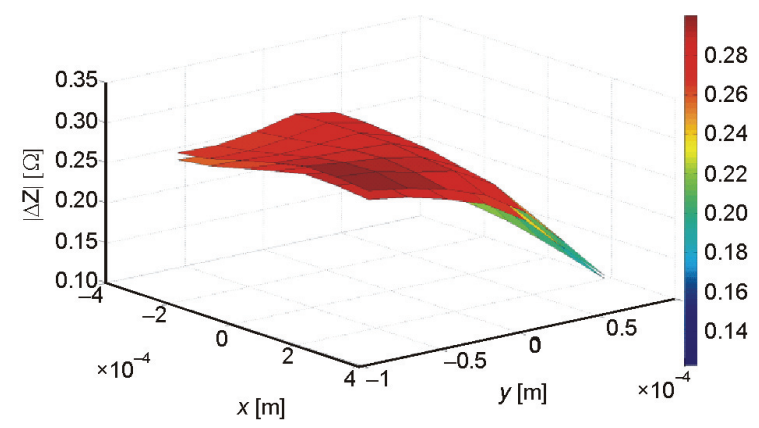

Fig. 12. Comparison of the target impedance magnitude with that obtained at the MM optimal point in case of cubical defect (crack 2)

Table 3. Inversion results for the MM optimization of investigated crack

\begin{tabular}{c|c|c|c|c|c|c}
\hline $\begin{array}{c}\text { Name of } \\
\text { defect }\end{array}$ & $\begin{array}{c}\text { Initial point } \\
{[\mathrm{mm}]}\end{array}$ & $\begin{array}{c}\text { MRE for } \\
\mathbf{x}_{c}[\%]\end{array}$ & $\begin{array}{c}\text { No of coarse } \\
\text { model } \\
\text { evaluation }\end{array}$ & $\begin{array}{c}\text { No of fine } \\
\text { model } \\
\text { evaluations }\end{array}$ & $\begin{array}{c}\text { Reconstructed } \\
\text { size of defect }\end{array}$ & $\begin{array}{c}\text { MRE for } \\
\mathbf{x}_{f}[\%]\end{array}$ \\
\hline $\begin{array}{c}\text { Crack 1 } \\
\text { (ellipsoidal } \\
\text { flaw) }\end{array}$ & {$\left[\begin{array}{c}0.20 \\
0.75 \\
0.375\end{array}\right]$} & $12 \%$ & 5 & 6 & {$\left[\begin{array}{l}0.32 \\
1.07 \\
0.49\end{array}\right]$} & $7.61 \%$ \\
\hline $\begin{array}{c}\text { Crack 2 } \\
\text { (cuboidal } \\
\text { flaw) }\end{array}$ & {$\left[\begin{array}{l}0.100 \\
0.375 \\
0.187\end{array}\right]$} & $14 \%$ & 3 & 4 & {$\left[\begin{array}{c}0.49 \\
1.98 \\
0.494\end{array}\right]$} & $6.7 \%$ \\
\hline $\begin{array}{c}\text { Crack 3 } \\
\text { (cylindrical } \\
\text { flaw) }\end{array}$ & {$\left[\begin{array}{l}0.100 \\
0.375 \\
0.187\end{array}\right]$} & $13 \%$ & 4 & 5 & {$\left[\begin{array}{l}0.287 \\
1.063 \\
0.447\end{array}\right]$} & $7.1 \%$ \\
\hline
\end{tabular}

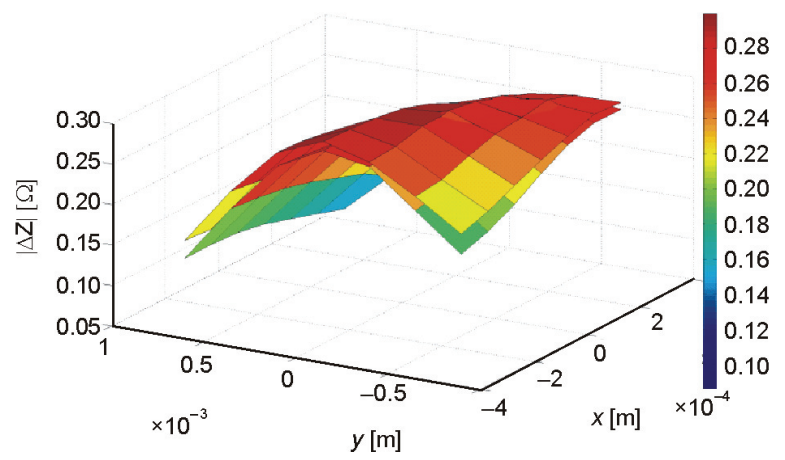

Fig. 13. Comparison of the target impedance magnitude with that obtained at the MM optimal point in case of ellipsoidal defect (crack 1) 


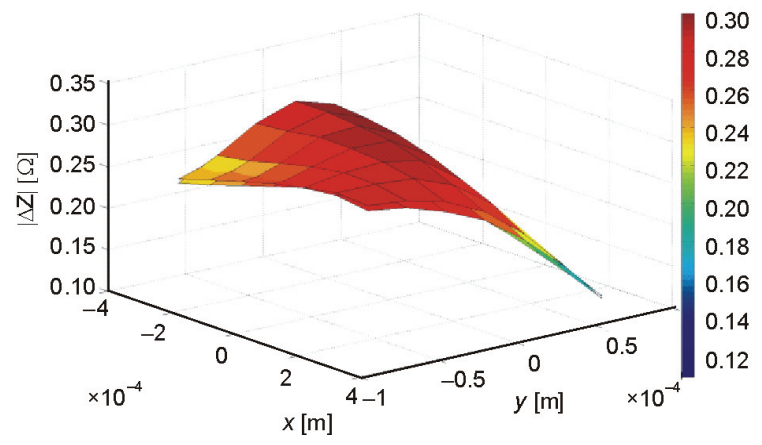

Fig. 14. Comparison of the target impedance magnitude with that obtained at the MM optimal point in case of ellipsoidal defect (crack 3)

The comparison between the target impedance and that calculated for the reconstructed shape of defects is shown in Figures 12, 13 and 14, respectively.

\subsection{Discussion}

Based on the results included in both tables one can conclude that the application of the SM or the MM methodology leads to a decrease in computational time for the estimation of the defects parameters using ECT data. The optimal solution is reached after only a few evaluation of time-consuming fine model.

Tables 2 and 3 show the good working of both minimization methods where for example in case of the elliptical defect 5 (6) evaluations need to be performed in the fine FE model and 5 (6) parameter extraction procedures, see equation (21) for the SM algorithm and equation (28) for the MM algorithm. In general, the MM technique need less evaluation of a fine model in order to obtain the comparable result of the defects reconstruction with respect to their accuracy. We save approximately $50 \%$ of CPU time when using the SM or MM algorithm, compared to the use of Tikhonov and $\operatorname{GCV}(\lambda)$ regularization in the fine FE model only. The reconstructed defects are accurate when using SM and MM. The MM is however able to recover the defect more accurately but the difference is small and negligible. The results presented in this paper show that the quality of the implemented coarse model relatively to the fine model is satisfactory. As shown in Figure 9 there is not a large misalignment between the parameter spaces of the coarse and fine model, i.e. the shift in parameter space between both models is relatively small. This explains the convergence of the SM method. In the response space, a misalignment exists which explains the more accurate convergence of the MM method compared to the SM method. Indeed, it is difficult for the SM method to deal with misaligned models in response space. However, we need to stress that the difference between MM and SM is still small. Both methods are thus suitable for recovering defects using the models presented in this paper. In order to further improve the accuracy of ECT, eddy current array technology, can be used. 


\section{Conclusion}

We investigated in this paper the eddy current inverse problem which relied on the parameters estimation of the surface slot located in a conductive plate made of Inconel 600. In order to accelerate the process of reconstruction based on the time-consuming FEM model, we applied the two-level techniques ASM and MM optimization. According to this methodology, a suitable coarse model is needed. In our case, for the purpose of 3D defect reconstruction, the reduced VIM approach was applied. Furthermore, the proposed algorithm was tested for varying shapes of defects. In all considered cases we achieved a proper result for defect parameters estimation, where the synthetic data was used as an input data. According to our experience, the two-level inversion procedures allow to save up to $50 \% \mathrm{CPU}$ time in comparison with the optimization by means of regularized Gauss-Newton algorithm in the same FE model. In this work only the specific kinds of surface defects were considered. Therefore, the reconstruction of arbitrary shapes of defects when using real measurement data from ECT system can be treated in further research.

\section{Acknowledgment}

This work was supported by the following projects IUAP P6/21, GOA07/GOA/006, FWO G.0142.08. G. Crevecoeur is a postdoctoral researcher of the FWO, P. Putek is a postdoctoral researcher within the IUAP P6/21 ("Inverse problems and optimization in low frequency magnetism") supported by Belgian government ${ }^{2}$.

\section{References}

[1] Isakov V., Uniqueness and stability in multidimensional inverse problems. Inverse Problems 9: 579621 (1993).

[2] Yamamoto M., A mathematical aspect of inverse problems for non-stationary Maxwell's equations. Int. J. Appl. Electromagn. Mech. 8: 77-98 (1997).

[3] Monebhurrun V., Duchene B., Lesselier D., Three-dimensional inversion of Eddy Current Data for non-destructive evaluation of steam generator tubes. Inverse Problems 14: 707-724 (1998).

[4] Badics Z., Pavo J., Fast Reconstruction from Eddy Current Data. IEEE Transactions on Magnetics 34(5): 2823-2828 (1998).

[5] Tamburino A., Rubinacci G., Fast methods for quantitative eddy-current tomography of conductive materials. IEEE Trans. Magn. 42(206): 2017-2028.

[6] Pirani A., Ricci M., Specogna R. at al., Multi-frequemcy identification of defects in conducting media. Inverse Problem 24: 1-18 (2008).

[7] Gawrylczyk K.M., Putek P., Adaptive meshing algorithm for recognition cracks. COMPEL 23: 677684 (2004)

[8] Chady T., Enokizono M., Sikora R. at al., Natural crack recognition using inverse neural model and multi-frequency eddy current method. IEEE Transactions on Magnetics 37(4): 2797-2799 (2001).

[9] Sikora R., Baniukiewicz P., Reconstruction of cracks from eddy current signals using genetic algorithm and fuzzy logic. Review of progress in quantitative nondestructive evaluation. Springer Verlag pp. 775-782 (2005).

\footnotetext{
${ }^{2}$ Interuniversity Attraction Poles (IAP) Phase VI, 2007-2011, http://www.belspo.be/belspo/iap/index_en.stm
} 
[10] Bandler J.W., Cheng Q.S., Dakroury S.A. et al., Space mapping: the state of the art. IEEE Transactions on Microwave Theory and Techniques 52(1): 337-361 (2004).

[11] Encica L., Echeverr 1a D., Lomonova E. et al., Efficient optimal design of electromagnetic actuators using space mapping. Structural and Multidisciplinary Optimization 33(6): 481-491 (2007).

[12] Crevecoeur G., Sergeant P., Dupré L., Van de Walle R., Two-level response and parameter mapping optimization for magnetic shielding. IEEE Transactions on Magnetics 44(issue 2): 301-308 (2008).

[13] Amineh R.K., Koziel S., Nikolova N.K. et al., A Space Mapping Methodology for defect characterization from magnetic flux leakage measurements. IEEE Trans. on Magnetics 44(8): pp. 2058-2065 (2008).

[14] Takagi T., Ueseka M., Miya K., Electromagnetic NDE research activities in JSAEM. Studies in Applied Electromagnetic and Mechanic vol. 12, IOS Press (1997).

[15] Biro O., Richeter R., CAD in elektromagnetism, advances in electronics and electron physics, P.W. Hawkes Ed 82 (1991).

[16] Bowler J.R., Jenkins S.A., Validation of three dimensional Eddy-Current probe flaw interaction model using analytical results. IEEE Transactions on Magnetics 26(5): 2085-2088 (1990).

[17] Norton S.J., Bowler J.R. Theory of eddy current inversion. Journal of Applied Physics 73: 501-513 (1993).

[18] Bowler J.R., Eddy current interaction with an ideal crack. Part I: The forward problem. Journal of Applied Physics 75(12): 8128-8137 (1994).

[19] Dodd C.V., Deeds W.E., Analytical solution to eddy-current probe-coil problem. Journal of Applied Physics 39(6): 2829-2838 (1968).

[20] Silvester P.P., Ferrari R.L. Finite elements for electrical engineers. Cambridge University Press, Cambridge, UK (1990).

[21] Bowler J.R., Jenkins S.A., Sabbagh L.D., Sabbagh H.A., Eddy-current impedance due to a volumetric flaw. Journal of Applied Physics 70(3): 1107-1114 (1990).

[22] Bowler J.R., Norton S.J., Harrison D.J. Eddy current interaction with an ideal crack. Part II: The inverse problem. Journal of Applied Physics 75(12): 8138-8144 (1994).

[23] Theodoulidis T.P., Kriezis E.E., Eddy current canonical problems (with applications to nondestructive evaluation). TechScience Press (2006).

[24] Monebhurrun V., Lesselier D., Duchene B., Evaluation of a 3-D bounded defect in the wall of a metal tube at eddy current frequencies: the direct problem. J. Electromagn. Waves Applic. 12: 315-347 (1998).

[25] Felipe J., Abascal P.J., Lambert M., 3-D eddy-current imaging of metal tubes by gradient-based controlled evolution of level sets. IEEE Trans. on Magnetics 44(12): 4721-4729 (2008).

[26] Dyck D.N., Lowther D.A.. A method of computing the sensitivity of electromagnetic quantities to changes in material and sources. IEEE Trans. on Magn. 30(5): 3415-3418 (1994).

[27] Gawrylczyk K.M., Putek P., Multi-frequency sensitivity analysis of $3 d$ models utilizing impedance boundary condition with scalar magnetic potential. Advanced Computer Techniques in Applied Electromagnetics 30 (2008).

[28] Hansen Ch., Regularization tools for a Matlab package for analysis and solution of discrete illposed problems. Numerical algorithm 6, the last version September pp.: 1-35 (2001).

[29] Echeverr' 1a D., Lahaye D., Encica L. et al., Manifold-mapping optimization applied to linear actuator design. IEEE Transactions on Magnetics 42(2): 1183-1186 (2007).

[30] Putek P., Crevecoeur G., Slodička M. et al., Application of space mapping methodology to defects recognition in eddy current testing. Proceedings of the 11th Workshop on Optimisation and Inverse Problems in Electromagnetism (OIPE), Sofia, Bulgaria, pp. P17 (2010).

[31] Koziel S., Bandler J.W., Madsen K. A space mapping framework for engineering optimization: theory and implementation. IEEE Trans. Microwave Theory Tech. 54(10): 3721-3730 (2006)

[32] Koziel S., Bandler J.W. Space mapping optimization with adaptive surrogate model. IEEE Trans. Microwave Theory Tech. 55(3): 541-547 (2007).

[33] Theodoulidis T., Poulakis N., Dragogias A. Rapid computation of eddy current signals from narrow cracks. NDT\&E International 43: 13-19 (2010). 